Preprint submitted to

Acta Phys. Hung. A 22/1 (2005) 000-000

HEAVY ION

PHYSICS

\title{
Identification of exotic jet topologies via three particle correlations in PHENIX
}

\author{
N. N. Ajitanand ${ }^{1}$ \\ ${ }^{1}$ Dept. of Chemistry, SUNY Stony Brook, \\ Stony Brook, NY 11794, USA
}

Received 10 November 2005

\begin{abstract}
Modifications of jet properties resulting from the coupling of jets to the strongly interacting matter produced in RHIC collisions are of great current interest. In recent work, the PHENIX collaboration has applied a novel technique to the analysis of two particle azimuthal correlations which extinguishes the harmonic part of the underlying event revealing the true jet shape. Recent extensions of the method to three particle correlations allow for a more revealing study of jet topologies in $\mathrm{Au}+\mathrm{Au}$ collisions at $\left(\sqrt{s_{\mathrm{NN}}}=200 \mathrm{GeV}\right)$.
\end{abstract}

Keywords: Jets, jet modification, three particle correlations, sonic boom PACS: see: http://www.aip.org/pacs/

\section{Introduction}

The energy density achieved in $\mathrm{Au}+\mathrm{Au}$ collisions at RHIC far exceeds the lattice QCD estimate for creating the QGP. The high matter density gives rise to large pressure gradients which are the driving force for the observed large azimuthal anisotropy $\left(v_{2}\right)$ of particle emission from the collision zone. The value of this anisotropy is close to the predictions of the hydrodynamic model which in turn implies the creation of a strongly interacting medium which undergoes early thermalization [1]. Jets provide good probes of this medium provided one can decompose the jet signal from the collective flow effects. Possible medium associated modifications of the jet topology are a conical emission due to a "sonic boom" effect [2] and deflection induced by interactions with the partonic flow [3]. Two and three particle azimuthal correlations can be an effective tool in the study of jet topology. 


\section{Two Particle Azimuthal Correlations}

To study jet topologies we use two- and three-particle azimuthal correlation functions. For two-particle correlations, the correlation function $C(\Delta \phi)$ is given by $C(\Delta \phi)=\frac{N_{\text {real }}(\Delta \phi)}{N_{\text {mix }}(\Delta \phi)}$, where $\Delta \phi$ is the difference of the azimuthal angles of the pair. The real distribution $\left(N_{\text {real }}(\Delta \phi)\right)$ is built from pair members belonging to the same event and the mixed distribution $\left(N_{m i x}(\Delta \phi)\right)$ is made of pair members belonging to different events. Thus the correlation function is free of geometric acceptance effects and carries only the combined correlations from flow and jets. Decomposition of these correlations into their jet and flow contributions, constitute an important prerequisite for obtaining the jet function and hence, information about jet fragmentation.

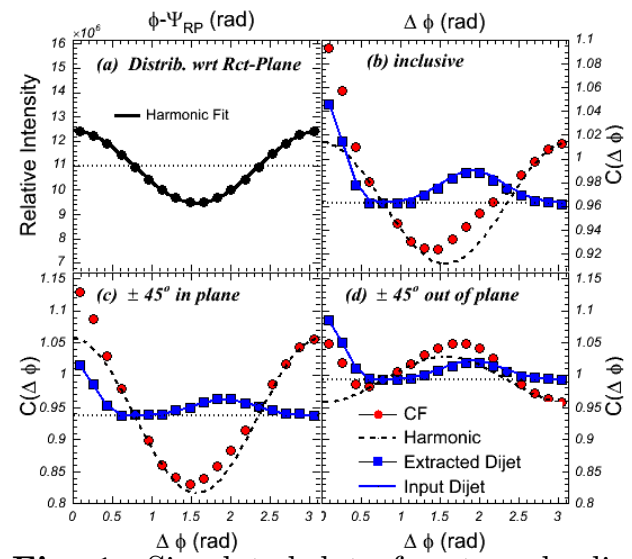

Fig. 1. Simulated data for strongly distorted away-side jets. The squares show retrieved points compared to the solid curves for the input jet functions.

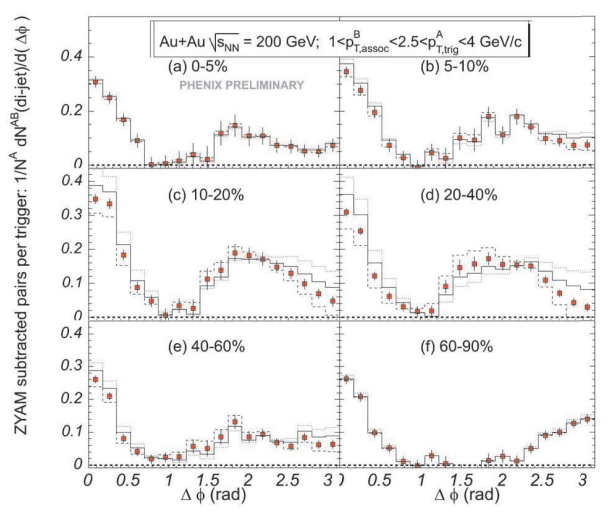

Fig. 2. Experimental jet-pair distributions [after flow subtraction] for several centrality selections.

Figure 11 show results from simulations in which strongly distorted away-side jets were studied. Panels (b), (c), and (d) show cases for inclusive, in-plane and out-of-plane correlation functions. The figure clearly shows that our decomposition method retrieves the input jet function in detail, confirming that the decomposition procedure is robust even for unusual di-jet distributions. Figure 2 show results obtained from the decomposition of the two-particle correlation measurements. The apparent shape distortions of the away-side jet is consistent with recent conjectures of a strong coupling between such jets and the high energy density matter that they traverse [2, 3].

\section{Three Particle Azimuthal Correlations}

Three-particle correlation functions consisting of a trigger hadron from the range $2.5<p_{T}<4.0 \mathrm{GeV} / \mathrm{c}$ (hadron \#1) and two associated hadrons from the range 
$1.0<p_{T}<2.5 \mathrm{GeV} / \mathrm{c}$ (hadron \#2 and \#3) were also studied (cf. Fig. ??). Correlation surfaces were constructed by way of $\Delta \phi_{1,2}$ and $\Delta \phi_{1,3}$ distributions. The correlation surface $C\left(\Delta \phi_{1,2}, \Delta \phi_{1,3}\right)=\frac{N_{\text {real }}\left(\Delta \phi_{1,2}, \Delta \phi_{1,3}\right)}{N_{\operatorname{mix}}\left(\Delta \phi_{1,2}, \Delta \phi_{1,3}\right)}$. Here $\Delta \phi_{1,2}$ and $\Delta \phi_{1,3}$ are the azimuthal angle difference between trigger and associated particle pairs. The mixed distributions were made of pair members belonging to different events. Consequently, the correlation functions contain both triples and doubles contributions.

One can extinguish the harmonic contributions to the correlation function by aligning the high $p_{T}$ particle perpendicular to the reaction plane followed by adjustment of a constraint "byte-angle" $\phi_{c}$, to achieve extinction [4];

$v_{2}^{\text {out }}(\operatorname{trig})=\left(\frac{2 v_{2}\left(\Delta \phi_{c}\right)-\sin \left(2 \Delta \phi_{c}\right)\left\langle\cos \left(2 \Delta \Psi_{R}\right)\right\rangle+\frac{v_{2}}{2} \sin \left(4 \Delta \phi_{c}\right)\left\langle\cos \left(4 \Delta \Psi_{R}\right)\right\rangle}{2\left(\Delta \phi_{c}\right)-2 v_{2} \sin \left(2 \Delta \phi_{c}\right)\left\langle\cos \left(2 \Delta \Psi_{R}\right)\right\rangle}\right)$.

\section{Results}

Simulated three-particle correlation surfaces $\left(\Delta \phi_{1,2}\right.$ vs. $\left.\Delta \phi_{1,3}\right)$ are shown in Figs. [3]5 for three distinct away-side jet scenarios; (i) a "normal jet" in which the away-side jet axis is aligned with the leading jet axis with a spread, (ii) a "deflected jet" in which the away-side jet axis is misaligned by $\sim 60^{\circ}$, and (iii) a "Cherenkov or conical jet" in which the leading and away-side jet axes are aligned but fragmentation is confined to a very thin hollow cone with a half angle of $\sim 60^{\circ}$. The simulated results show relatively clear distinguishing features for the three scenarios considered.

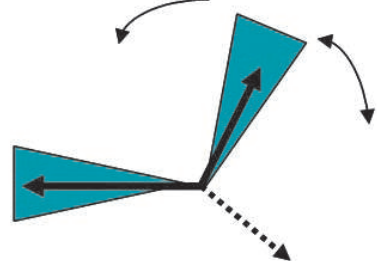

\section{Deflected jet}

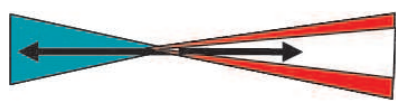

\section{Cherenkov jet}

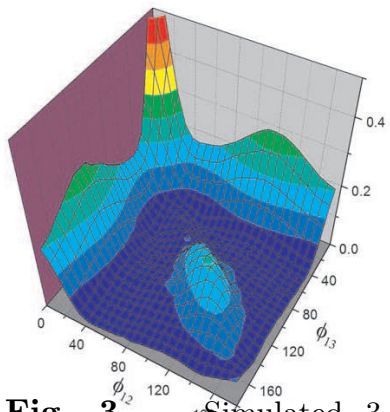

Fig. 3. ${ }^{2}$ की 120 imulated 3particle correlations for "deflected" jets.

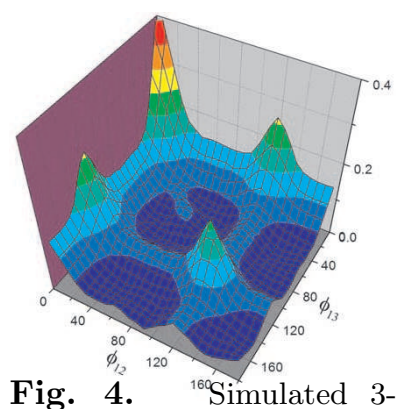

particle correlations for conical flow.
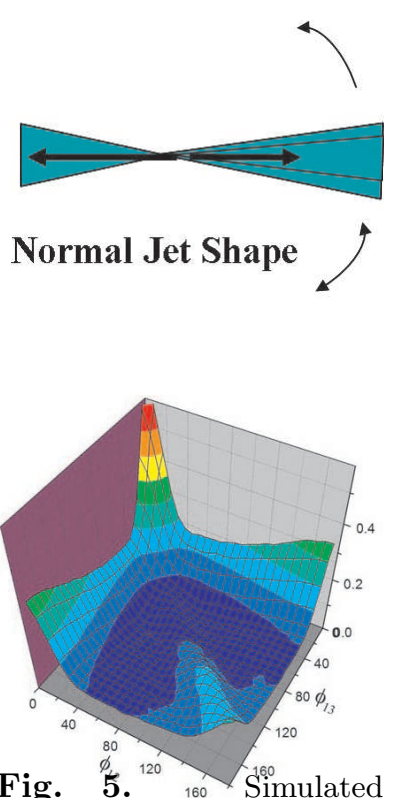

3-particle correlations for "normal" jets. 


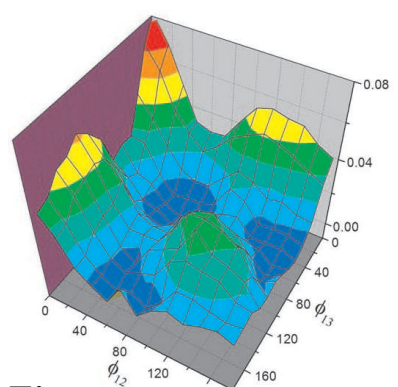

Fig. 6. Hædron-hadron-

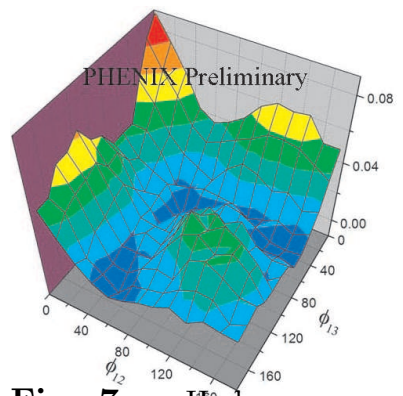

Fig. 7. Hadron-meson-

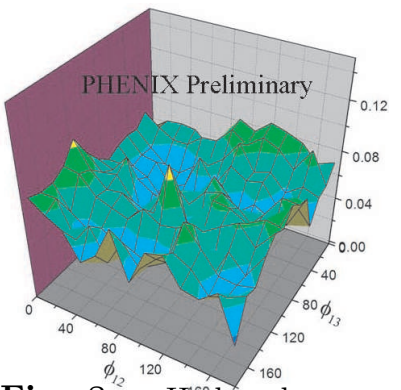

Fig. 8. Hisodron-baryonhadron correlation function. meson correlation function baryon correlation function. are shown in Figs. 6]-8 They show a strong dependence on the flavor (PID) of the associated particle and clearly do not follow the expected patterns for a "normal jet". We conclude that these three-particle correlation surfaces provide additional compelling evidence for strong modification of the away-side jet. Further detailed quantitative investigations are however required to firm up the signatures in the data which distinguish between a "deflected jet" and a "Cherenkov jet".

\section{Summary}

Two and three particle correlation functions have been analyzed to extract the awayside jet structure for a high $p_{T}$ trigger-particle in association with low $p_{T}$ particles. Jet landscapes and jet-pair distributions have been obtained as a function of event centrality and particle flavor. Preliminary comparisons to simple simulations do not exclude "Mach cone" like features in the data.

\section{References}

1. R. Lacey, nucl-ex/0510029.

2. Casalderrey-Solana,Shuryak, Teaney hep-ph/0411315

3. Armesto, Salgado, Wiedemann, hep-ph/0411341

4. Ajitanand Alexander, Chung, Holzmann, Issah, Lacey, Shevel, Taranenko P. Danielewicz Phys.Rev. C 72, 011902 (2005)

5. Bielcikova,Esumi,Filimonov, Voloshin,Wurm Phys. Rev. C 69, 021901 (2004)

6. K. Adcox et al, PRL 87, 052301 (2001). 